\title{
ACCESIBILIDAD DE LA POBLACIÓN A LAS AGLOMERACIONES URBANAS DE LA PENÍNSULA IBÉRICA
}

\author{
J. A. Gutiérrez Gallego ${ }^{1}$ \\ Mora Aliseda ${ }^{2}$ \\ GÓmeZ DOMÍNGUEZ ${ }^{2}$ \\ JARAÍZ CABANILLAS ${ }^{2}$
}

\begin{abstract}
Resumen - Con anterioridad al desarrollo de las infraestructuras el espacio era continuo, ya que los tiempos de acceso aumentaban gradualmente con la distancia. En el transporte por carretera, las autopistas y autovías han producido una contracción del espacio, al reducir las distancias en tiempo. Pero esta contracción no ha sido uniforme para todo el espacio. Los nodos de unión de las principales vías que suelen corresponder con las aglomeraciones urbanas, son los principales beneficiarios del nuevo orden espacial, al quedar conectados entre sí de forma rápida y eficiente. Esto lleva a considerar una visión distinta a los desequilibrios centro-periferia: los desequilibrios entre las principales aglomeraciones urbanas y su entorno regional, característicos de ese espacio cada vez más discontinuo.

Por consiguiente, las nuevas infraestructuras de transporte, esenciales para el desarrollo socioeconómico y la articulación e integración de municipios y espacios, pueden modificar la accesibilidad y la dinámica poblacional influyendo en el desarrollo regional. Se pretende pues con el estudio, analizar la relación existente entre la accesibilidad y el desarrollo de las infraestructuras de transporte en las principales aglomeraciones urbanas de la Península Ibérica.
\end{abstract} nas, SIG.

Palabras clave: Infraestructuras, transporte, accesibilidad, aglomeraciones urba-

Abstract - ACCESSIBILITY OF THE POPULATION TO THE URBAN AGGLOMERATIONS of the Iberian Peninsula. Prior to the development of infrastructure, space had a continuous character, given that the time it took to access a given place increased gradually with the distance to that place. Motorways and state highways have produced a contraction of space as far as road transport is concerned, due to the decrease in distance as measured in terms of time. However, this contraction has not been uniform across space. The main beneficiaries of the new spatial order are the nodes that link together trunk roads - which usually coincide with urban agglomerations -, insofar as they are connected to each other in quick and efficient ways. This gives rise to a

Recebido: 08/04/2008. Aceite: 07/05/2009.

1 Centro Universitario de Mérida. Universidad Extremadura. E-mail: jagutier@unex.es

2 Facultad de Filosofía y Letras. Universidad Extremadura. E-mail: jmora@unex.es; ameba_52@hotmail.com; jfjaraiz@unex.es 
new way of looking at the imbalances between the centre and the periphery - that is to say, between the main urban agglomerations and their regional hinterlands, as structured in an increasingly discontinuous space. As a consequence, the construction of new transport infrastructures (which are essential to socioeconomic development and to the articulation and integration of the various spaces and localities) can modify the population's spatial dynamics and accessibility levels, with crucial consequences in terms of regional development. With this in mind, this study proposes to analyse the current relationship between the development of transport infrastructures and accessibility in the case of the main urban agglomerations in the Iberian Peninsula.

Keywords: Infrastructure, transport, accessibility, urban agglomerations, GIS.

Resumo - ACESSIBILIDADE DA POPULAÇ̃̃O AOS PRINCIPAIS NÚCLEOS URBANOS DA Península IbÉRICA. Antes do desenvolvimento das infra-estruturas o espaço era contínuo, no sentido em que os tempos de percurso aumentavam gradualmente com a distância. No transporte por estrada, as auto-estradas e outras vias rápidas produziram uma contracção do espaço, ao reduzirem as distâncias em tempo. Mas esta contracção não foi uniforme para todo o espaço. Os nós de uniões das principais vias que estabelecem correspondência com as aglomerações urbanas são os principais beneficiários da nova ordem espacial, ao ficarem conectados entre si de forma rápida e eficiente. Isto permite uma visão distinta dos desequilíbrios centro-periferia: os desequilíbrios entre as principais aglomerações urbanas e o seu enquadramento regional, característicos desse espaço cada vez mais descontínuo.

Por conseguinte, as novas infra-estruturas de transporte, essenciais para o desenvolvimento socioeconómico e a articulação e integração de municípios e espaços, podem modificar a acessibilidade e a dinâmica populacional influindo no desenvolvimento regional. Com o estudo, pretende-se analisar a relação existente entre a acessibilidade e o desenvolvimento das infra-estruturas de transporte nas principais aglomerações urbanas da Península Ibérica.

Palavras-chave: Infra-estruturas, transporte, acessibilidade, aglomerações urbanas, SIG.

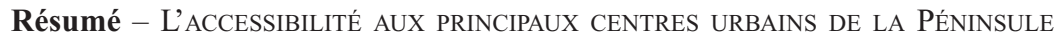
IBÉRIQUE. Avant le développement des infrastructures routières, l'espace pouvait être considéré comme continu, puisque les temps de parcours augmentaient graduellement avec la distance. L'apparition des autoroutes et autres voies rapides a provoqué une contraction spatiale, par réduction de l'espace-temps, mais de façon non uniforme. Les grands carrefours périurbains ont été les principaux bénéficiaires du nouvel ordre spatial, puisqu'ils sont interconnectés de façon rapide et efficace. En fonction de ce fait, on doit donc revoir la notion centre-périphérie, c'est-à-dire les rapports liant les principaux centres urbains à leur cadre régional, dans un espace de plus en plus discontinu. Les nouvelles infrastructures de transport - essentielles pour le développement socio-économique et pour l'articulation et l'intégration des unités administratives -, modifient l'accessibilité et la répartition de la population, en influençant ainsi le développement régional. On a tenté d'analyser ce problème relativement aux principaux centres urbains de la Péninsule Ibérique.

Mots-clés: Infrastructures, transports, accessibilité, centres urbains, SIG. 


\section{ANTECEDENTES}

La relación entre las infraestructuras de transporte y el desarrollo regional provoca dos efectos distintos: un efecto no espacial que es aquel que se obtiene al agregar inversiones en infraestructura sobre la actividad económica, la producción y la competencia en una economía; y un efecto espacial en el que la infraestructura es el elemento que da lugar a los diferentes comportamientos en las diferentes localizaciones, entre dos regiones cualquiera o dentro de la misma (Vickerman, 1995). El aumento de infraestructuras de transportes por sí solo no garantiza el desarrollo regional; aspectos como la mano de obra especializada o la situación geoestratégica son complementos muy importantes para conseguir el crecimiento deseado. Biehl (1986) señala cuatro factores determinantes del desarrollo regional:

Infraestructuras: Entendidas en el sentido amplio, engloba no sólo a las infraestructuras básicas (transporte, agua, electricidad, etc.), sino también a los equipamientos (universidades, hospitales, etc.).

Localización: Hace referencia a la lejanía o proximidad de una región respecto a los principales centros de actividad económica.

Aglomeración y estructura de los asentamientos: Refleja la concentración espacial de la población, de los productores y de los consumidores en el interior de una determinada región.

La estructura sectorial: Se refiere a la relación entre el volumen relativo de los sectores de la agricultura, la industria y los servicios, por una parte, y el nivel de desarrollo en términos de ingresos per cápita, por otra.

Históricamente los planes territoriales de infraestructuras se basaban principalmente en ampliar y mejorar las vías existentes, en la actualidad se observa necesario un replanteamiento en el que, además de continuar con estas ampliaciones y mejoras, se ponga en marcha una nueva vertebración del territorio, más homogéneo y equilibrado que mejore la accesibilidad de aquellas regiones que a lo largo de la historia han estado peor dotadas de infraestructuras.

Las políticas de transporte interregional deben tener en cuenta que como media los costes de transporte representan el 2\%-3\% del coste de la producción total, lo cual indica que una mejora en la accesibilidad no va a asegurar un cambio en la localización de la empresa, ya que tienen mayor protagonismo otros aspectos como la especialización de la mano de obra, el clima social, el nivel de los salarios o los incentivos regionales a la inversión privada. Esto quiere decir que una inadecuada accesibilidad va a complicar mucho el asentamiento de actividades económicas en una zona, pero tampoco unas buenas condiciones van a asegurar dicho asentamiento.

Debido a que un cambio de localización de una empresa es caro y arriesgado, las mejoras en infraestructuras y accesibilidad suelen ser insuficientes para provocar ese cambio de ubicación. En este sentido, las políticas de transporte interregional si verdaderamente buscan un desarrollo regional deben incluir además actuaciones territoriales: creación de zonas de actividad, ayudas financieras y fiscales, promoción del turismo, operaciones de ordenación urbana y operaciones inmobiliarias, promoción de las ciudades afectadas, modificación de los servicios de viajeros urbanos, centros de carga y zonas de actividades logísticas, etc. 
Las actuaciones dentro del sector económico del transporte siempre se han encontrado con la disyuntiva de cuáles son las zonas prioritarias en las que desarrollar las infraestructuras, teniendo en cuenta que no existen recursos para todos los sitios a la vez, ¿debe ser éste un instrumento sectorial de redistribución de rentas o deben prevalecer las políticas fiscales y de gasto público?, en otras palabras, ¿debe servir para desarrollar aquellas zonas más deprimidas, teniendo en cuenta la importancia que tiene el desarrollo de las infraestructuras, o se deben mejorar las condiciones de aquellas zonas más desarrolladas para evitar la congestión ya que son los que hacen un mayor uso de las infraestructuras? Haggett (1975) afirma que se debe favorecer el mayor número posible de usuarios y mejorar el equilibrio entre ellos.

La relación existente entre transporte, accesibilidad y usos del suelo también es bastante clara. Las infraestructuras de transporte proporcionan lugares con una determinada accesibilidad, la cual influye sobre los usos del suelo. Una mayor inversión en infraestructuras modifica la accesibilidad de una determinada área. Reduciendo el tiempo de viaje entre los orígenes y destinos, conectados por estas nuevas infraestructuras, estos puntos llegan a ser más accesibles en relación con otros orígenes y destinos en el área. Por consiguiente, se puede atraer nuevas actividades a esas áreas como resultado del incremento del nivel de accesibilidad, algunas de la cuales podían haber cambiado su ubicación en aras de una mejor localización (Giuliano, 1986). Una mayor densidad residencial también resulta de presiones del mercado que hacen un uso más intensivo del suelo accesible. Así, normalmente, una mayor densidad residencia es la consecuencia, no la causa, de una mayor accesibilidad (Gutiérrez y Gómez, 1999).

También en opinión de los profesores Gutiérrez y Gómez (1999), la accesibilidad tiene un valor social y económico tanto individual como empresarial. El valor de la accesibilidad, los niveles o valores más o menos elevados, estarían lógicamente relacionados con la calidad de vida, la satisfacción, el interés y el desarrollo económico. Apuntan que existe también una relación, aunque compleja, entre la accesibilidad y el coste social y económico del viaje. Todo esto, con independencia de la importancia que tiene ya de por si la accesibilidad para comprender, pronosticar e influir en los modelos de localización urbana y usos del suelo (Helling, 1996).

\section{OBJETIVOS}

La principal pretensión de este trabajo es analizar la accesibilidad por carretera a lo que se ha considerado como aglomeraciones urbanas dentro del marco de la Península Ibérica.

En el caso de España, dichas aglomeraciones son aquellas áreas contiguas, formadas por uno o varios municipios, en la que la suma de la población potencial residente en un determinado tiempo y a un radio medio de distancia al núcleo central supera, en el último año de referencia, los 200.000 habitantes o queda muy cercana a ella.

En Portugal este umbral de población sería elevado, ya que de este modo sólo se englobarían las grandes aglomeraciones de este país. Por consiguiente, se ha considerado un umbral de 100.000 habitantes, de tal manera que se consideren no sólo dichas aglomeraciones, sino también las ciudades con mayor área de influencia, entidad poblacional y capacidad de mercado. 


\section{MATERIAL Y MÉTODOS}

Para la elaboración de este artículo se ha creado a través de un sistema de información geográfica, SIG, un modelo de accesibilidad a las principales aglomeraciones urbanas peninsulares, basado en tres indicadores de accesibilidad diferentes: indicador de accesibilidad absoluta, accesibilidad relativa y tiempos mínimos. Para cada indicador ha sido realizado un mapa, el cual se complementa, para su correcta interpretación, con un histograma de frecuencias en el que se muestra la distribución de los núcleos de población de la Península Ibérica.

En este estudio es necesario formular, por un lado, un modelo de transportes basado en la teoría de transportes que trate de aproximarse al modelo real todo lo posible y, por otro, hay que aplicar las herramientas adecuadas de los sistemas de información geográfica para materializar este modelo y de esta forma poder estudiarlo.

En cuanto al modelo teórico hay que hacer dos apreciaciones: en primer lugar, el sistema de transportes regional es un sistema cerrado y en segundo, los núcleos de población se consideran puntuales, sin superficie.

Los procedimientos seguidos para la formación del SIG corresponden a la captura de la información y el tratamiento de la misma, obteniendo posteriormente diferentes mapas para su análisis:

- Captura de la información: obtención de la cartografía; estructura de los datos.

- Tratamiento de la información: creación de topología; cálculo de las propiedades geométricas de la red; determinación de la impedancia de los tramos de la red de carreteras.

- Análisis: determinación de los caminos óptimos; cálculos de indicadores.

\section{CONCEPTOS SOBRE AGLOMERACIONES URBANAS}

En su Dictamen de 14 de mayo de 1998 sobre la "Comunicación de la Comisión: Hacia una política urbana para la Unión Europea”, el comité de las regiones desarrolló el concepto de región urbana funcional para describir a la metrópolis y su área de influencia. En éste se reconoce que las ciudades se transforman primero en conurbación y después en metrópolis, y que a continuación se extienden para formar regiones urbanas.

El proyecto europeo Urban Audit ${ }^{3}$ distingue tres niveles geográficos: la ciudad centro, la zona urbana más amplia (LUZ) y el barrio infraurbano. De acuerdo con dicho proyecto, la LUZ corresponde aproximadamente a la región urbana funcional. Al igual que este proyecto, el programa $\mathrm{ESPON}^{4}$ define la región urbana funcional como el área comprendida entre el área urbana o municipio principal y la franja de municipios adyacentes. Esta región urbana funcional será, por tanto, lo que hemos definido como aglomeración urbana.

3 Proyecto europeo donde se recoge información sobre las condiciones de vida en 258 ciudades de tamaño mediano y grande dentro de la Unión Europea y los países candidatos (UE-27).

4 Observatorio en Red de la Ordenación Territorial Europea. Programa de investigación de cinco años que compila y compara los datos sobre las tendencias del desarrollo en toda Europa. 
Para entender el significado de aglomeración urbana por completo, es necesario mencionar el concepto de metápoli descrito por Ascher. Una metápoli es el conjunto de los espacios para los cuales todo o una parte de sus habitantes, de sus actividades económica y territorios están integrados en el funcionamiento cotidiano (ordinario) de una metrópoli. Una metápoli implica, generalmente, una sola cuenca de empleo, de hábitat y de actividades. Los espacios que componen la metápoli son profundamente heterogéneos y no necesariamente contiguos. Una metápoli comprende, por lo menos, unos centenares de miles de habitantes (Ascher, 1995).

Tomando como referencia el modelo representado en la figura 1, lo que se ha calculado es la población potencial existente a menos de 30 minutos de cada uno de los núcleos de población mayores de 1.000 habitantes en la Península Ibérica. Este tipo de cálculo dinámico podría filtrarse para cualquier tiempo o incluso para distintos niveles administrativos (provincias, comunidades autónomas o países).

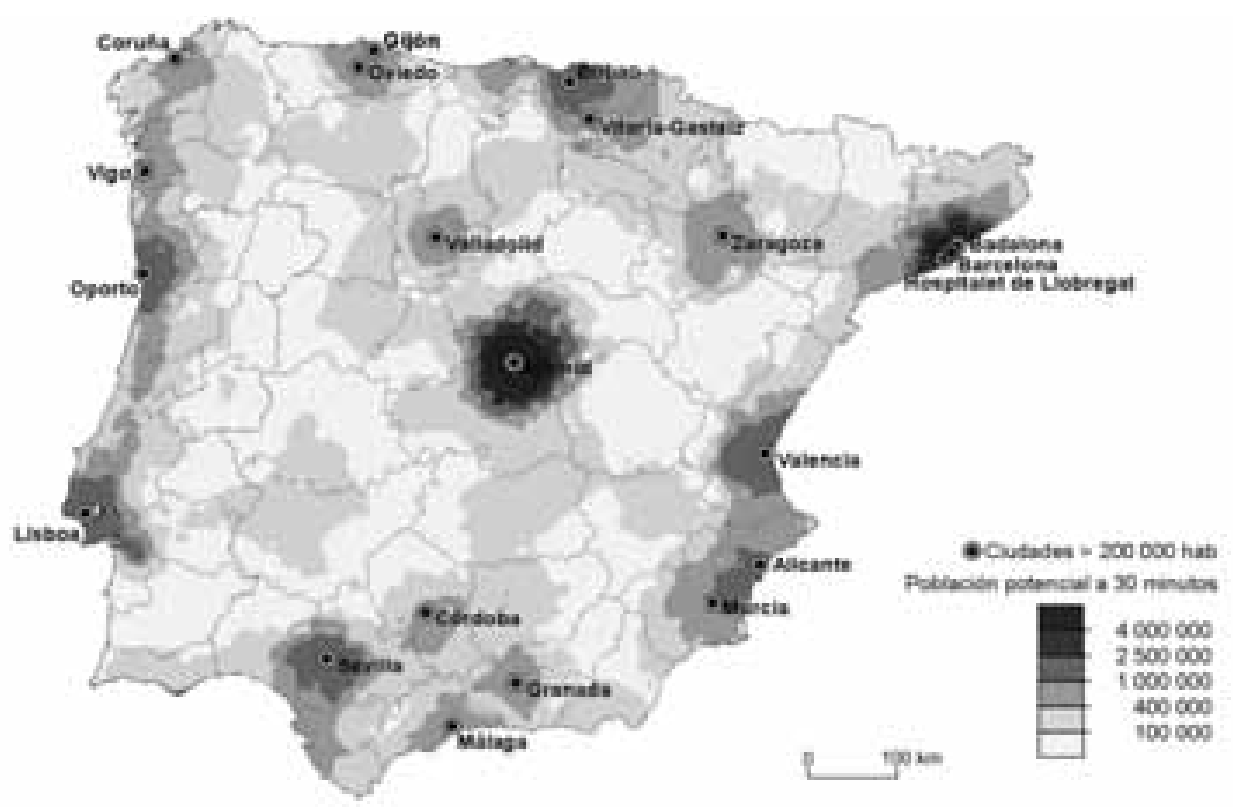

Fig. 1 - Población potencial a 30 minutos.

Fig. 1 - Potential population within a 30-minute access time.

Las manchas que podemos apreciar en la figura, a que da lugar el cálculo del potencial de población a 30 minutos de las ciudades con más de 200.000 habitantes, nos muestran las verdaderas aglomeraciones urbanas de la península y su entidad poblacional y espacial.

Como se comprueba, con este umbral de población para representar los potenciales de población se contempla una vasta red de ciudades medias y grandes de España pero no de Portugal, donde sólo se representan las principales aglomeraciones. Dado esta limitación, para el cálculo de los indicadores de accesibilidad estudiado a seguir se ha optado por definir un umbral menor para Portugal (100.000 habitantes) de manera que también se engloben las ciudades medias con una importante área de influencia y 
capacidad de mercado, llevando a cabo un análisis peninsular mucho más significativo y relevante.

\section{INDICADORES DE ACCESIBILIDAD}

La dificultad a la hora de definir los indicadores de accesibilidad fue apuntada ya por Pirie en 1979: "es posible que todo el mundo entienda la misma cosa por accesibilidad y simplemente resulte que puede ser medida de varias formas, o que debe ser medida de varias formas dadas las peculiaridades o limitaciones del problema".

Así pues, el concepto de accesibilidad ha sido muy complicado de definir y ha tenido varias acepciones: (i) en los primeros estudios se consideraba únicamente la distancia espacial entre dos puntos o con el resto del área de estudio; (ii) más recientemente se entiende como accesibilidad el beneficio neto que un grupo de personas obtiene por estar ubicados en una determinada localización y poder utilizar un sistema de transporte concreto; (iii) todos estos cambios en el concepto de accesibilidad, han dado lugar a que se hable de un concepto de distancia social en vez de distancia física (Monzón, 1988).

El fin último de los indicadores de accesibilidad no es solamente el análisis territorial y sus pronósticos de futuro, sino que tomarán sentido en el momento en que sirvan de base a la política de distribución de las ayudas económicas, contribuyendo de esta forma a alcanzar la cohesión económica y social que requiere el funcionamiento de la UE. Es por esto por lo que también se debería incorporar el concepto de accesibilidad entre los objetivos que las técnicas de análisis multicriterio suelen aplicar para la evaluación y selección de proyectos, siendo preciso, para ello, unificar los conceptos y criterios de determinación de los indicadores a utilizar en cada caso (Izquierdo y Monzón, 1992).

Se puede afirmar que la accesibilidad de una localización es muy importante para su desarrollo económico, social, cultural, urbanístico, etc.; por este motivo, se deben establecer unas correlaciones entre los indicadores de accesibilidad y las variables socioeconómicas y territoriales. En este sentido, el análisis de la accesibilidad puede convertirse en un elemento de prognosis (Izquierdo y Monzón, 1992).

\section{Accesibilidad absoluta}

La accesibilidad absoluta (IAA $A_{i}$ mide el grado de interconexión de un punto con el resto de puntos de la región estudiada, relacionando los potenciales de población y el tiempo mínimo a través de la red de los núcleos de población a las principales aglomeraciones urbanas. Se trata de calcular la media ponderada del tiempo mínimo que separa a cada núcleo de población con respecto a las diferentes aglomeraciones urbanas a través de la red, considerando la población de éstas como un factor de ponderación, según:

$$
I A A_{i}=\frac{\sum_{j=1}^{n}\left(I R_{i j} * R C A E_{j}\right)}{\sum_{j=1}^{n} R C A E_{j}}
$$

Donde $I R_{i j}$ es el tiempo mínimo entre los nodos $i j$, a través de la red, y $R C A E_{j}$ es la población de las principales aglomeraciones urbanas de la Península Ibérica. 


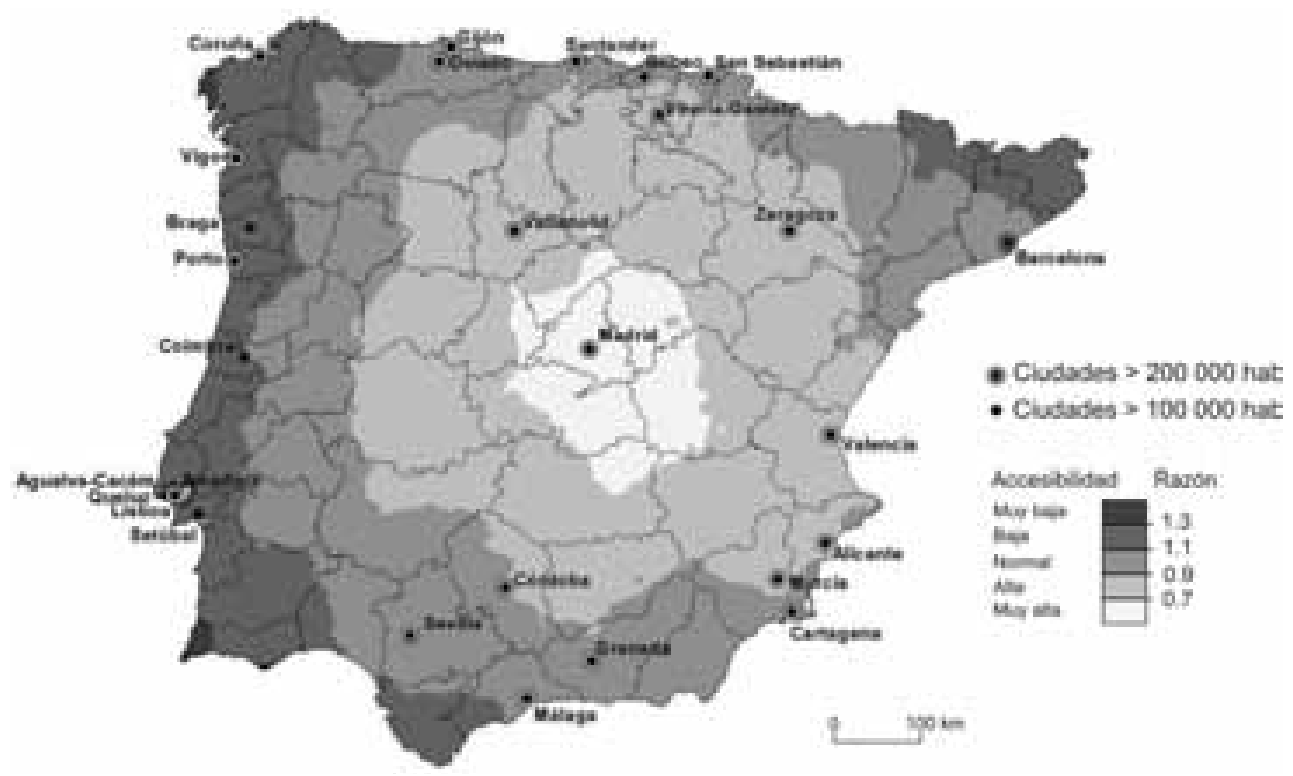

Fig. 2 - Accesibilidad absoluta.

Fig. 2 - Absolute accessibility.

La figura 2 representa claramente un modelo centro-periferia en el que Madrid, ubicado en el centro peninsular, es quien tiene una mayor accesibilidad absoluta, para ir descendiendo los valores de forma concéntrica, aunque desplazado hacia el este, conforme se analizan emplazamientos más alejados de la capital de España.

Las zonas con accesibilidad absoluta muy baja se reducen al suroeste de Portugal (distritos de Beja y Faro), y al extremo más occidental de la provincia de A Coruña en la Comunidad Autónoma de Galicia.

\section{Accesibilidad relativa}

La accesibilidad relativa $\left(\operatorname{IAR}_{\mathrm{i}}\right)$ a las principales aglomeraciones urbanas de la Península Ibérica es un indicador que muestra con gran nitidez el impacto de las infraestructuras sobre el territorio, debido a que neutraliza el efecto de la localización geográfica de los núcleos urbanos. Este índice cuantifica la calidad del trazado, analizando su grado de aproximación a la línea recta, la cual se entiende como el trazado ideal.

El índice de accesibilidad relativa compara el tiempo mínimo existente entre un núcleo urbano y las aglomeraciones urbanas con el tiempo ideal, entendiendo por tiempo ideal el que habría si se dispusiera de una unión en línea recta por medio de una autovía entre el núcleo urbano y la aglomeración urbana. Dicho indicador viene expresado por la siguiente formulación matemática:

$$
I A R_{i}=\sum_{j=1}^{n}\left(\frac{I R_{i j}}{I I_{i j}} \times R C A E_{j}\right)
$$


Donde:

$I R_{i j}$ es el tiempo mínimo entre dos puntos a través de la red de carreteras, $I I_{i j}$ la impedancia ideal (en línea recta) y $R C A E_{j}$ la población de las principales aglomeraciones urbanas de la península.

La impedancia ideal entre el nodo de origen y la aglomeración urbana destino, es la que se obtendría en línea recta por una infraestructura de gran calidad en el caso del transporte terrestre.

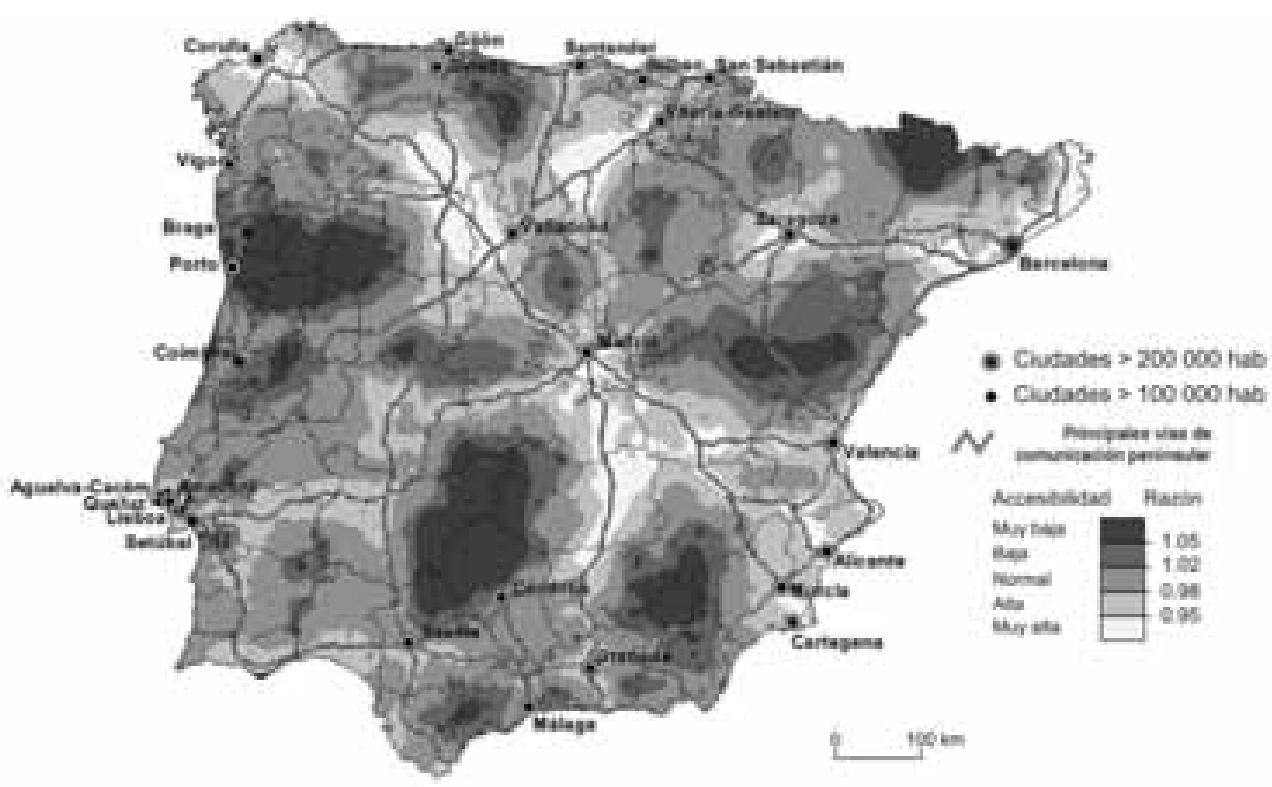

Fig. 3 - Accesibilidad relativa.

Fig. 3 - Relative accessibility.

En la figura 3 se representa el indicador de accesibilidad relativa para la Península Ibérica. Las principales vías de comunicación definen los valores de accesibilidad relativa en la Península Ibérica.

El mapa muestra claramente que los espacios existentes entre las principales vías de comunicación son los que tienen una accesibilidad relativa entre normal y muy baja, mientras que las parcelas de territorio por las que discurren las principales vías de comunicación poseen una accesibilidad normal, alta y muy alta.

Como se ha explicado al comienzo de este epígrafe, el indicador de accesibilidad relativa lo que pretende es calcular el trazado ideal, considerando que éste sería la línea recta. En algunos casos, principalmente en la mitad septentrional de Portugal y en zonas de Castilla y León y Extremadura, se puede ver que zonas cercanas a vías de comunicación importantes no muestran una accesibilidad alta; esto es debido a lo que se denomina índice de rodeo. Este índice suele ser elevado en zonas con una orografía sinuosa (montañas, ríos, grandes embalses, etc.), donde las comunicaciones no van más allá de carreteras autonómicas que tienen que rodear el accidente en cuestión. 


\section{Tiempos mínimos}

En la figura 4 se muestra el tiempo mínimo desde cualquier nodo de origen a la aglomeración urbana principal más cercana a él. Para esto se tiene en cuenta, además de la distancia, las vías por las que hay que transcurrir y su velocidad media; así pues, tanto la situación geográfica de los núcleos urbanos como las características de la red van a ser elementos muy importantes para los resultados finales de este indicador.

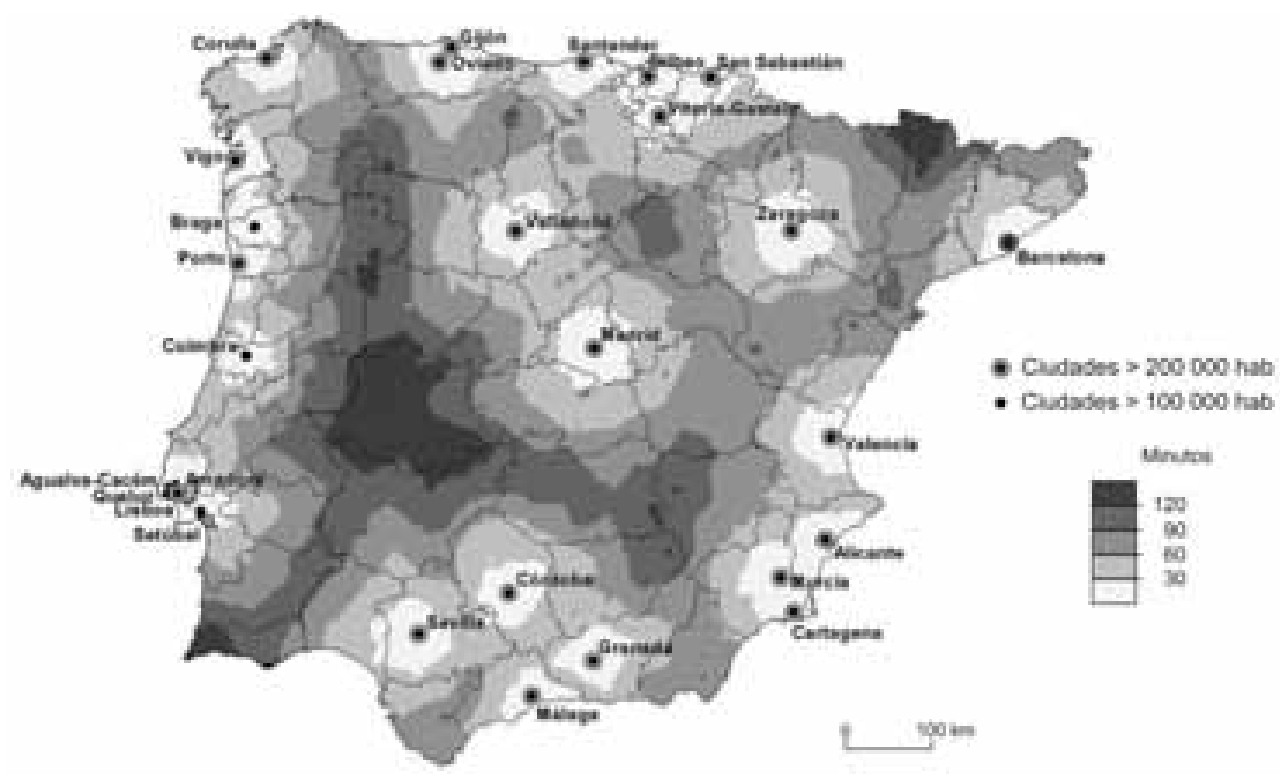

Fig. 4 -Tiempos mínimos de acceso a las aglomeraciones urbanas.

Fig. 4 - Minimum time required to access the urban agglomerations.

Las aglomeraciones urbanas más importantes de la península y los núcleos ubicados en su área de influencia se presentan como focos aislados e independientes dentro del territorio peninsular. Se ve como las principales aglomeraciones urbanas de la península actúan como focos de atracción económica y tienen un área de influencia bien definida a su alrededor, compuesta por infinidad de núcleos de población a menos de 60 minutos por carretera. Por consiguiente, la entidad poblacional y económica y las vías que comunican con cada una de estas ciudades, les van a conferir una mayor o menor área de influencia, es decir, un mayor o menor número de núcleos situados a menos de 60 minutos por carretera.

Conforme aumenta la distancia a estos núcleos de población, aparecen de forma concéntrica cambios en el color que caracteriza los tiempos mínimos de acceso en minutos; representando, a medida que el trayecto es mayor, un aumento en el tiempo mínimo de acceso. Los espacios existentes entre estas aglomeraciones, y fuera de su primera orla de núcleos que conforman su área de influencia, suelen presentar unos tiempos mínimos entre los 60 y los 90 minutos. 
En la Península Ibérica todavía se encuentran territorios y núcleos a más de dos horas de camino de la aglomeración urbana más cercana. Destaca sobremanera, gran parte del territorio extremeño, ante todo la mayor parte de la provincia de Cáceres, y el este del distrito de Castelo Branco en Portugal.

\section{CONCLUSIONES}

Una vez realizados y analizados los mapas resultantes de haber aplicado los diferentes indicadores de accesibilidad antes mencionados, se pueden llegar a las siguientes conclusiones:

- La red de carreteras actual aporta un modelo centro-periferia en lo referente a la accesibilidad absoluta de las principales aglomeraciones urbanas peninsulares; donde Madrid es la ciudad con mayor accesibilidad, teniendo la mejor conexión con las demás aglomeraciones urbanas.

- La totalidad del espacio transfronterizo, excluyendo el norte y un área reducida de la zona sur, se sitúa a más de 90 minutos de alguna aglomeración urbana principal. Por tanto, se debe seguir invirtiendo y potenciando el crecimiento económico y social de este territorio, el cual posee potencial suficiente para poder seguir desarrollándose alejado de los centros de decisiones.

- La entidad poblacional de A Coruña, Vigo y Oporto, su cercanía y las infraestructuras de gran capacidad que las conectan conforman una verdadera "euroregión" comercial con un intercambio constante de bienes, servicios e información.

\section{AGRADECIMIENTOS}

Este artículo se incluye en el proyecto de investigación realizado en el Centro Universitario de Mérida titulado ACCESIBILIDAD A LOS CENTROS DE ACTIVIDAD ECONÓMICA DE LA PENÍNSULA IBÉRICA, el cual se está desarrollando tras haber obtenido la concesión definitiva de subvención en la convocatoria de Ayudas a la Investigación del año 2005 en el Área de Transportes, promovida por el Ministerio de Fomento y publicada en el "Boletín Oficial del Estado" el 16 de diciembre del año 2005.

\section{BIBLIOGRAFÍA}

Ascher A (1995) Metapole ou L'avenir des villes. Odile Jacob, Paris.

Biehl D (1986) The contribution of infrastructure to the regional development. Final Report of the Infrastructure Study Group, Document, Commission of the European Communities, parts I and II, Office for the Official Publications of the European Communities. Luxemburgo.

COMITÉ ECONÓMICO Y SOCIAL EUROPEO (2007) Las áreas metropolitanas europeas: repercusiones socioeconómicas para el futuro de Europa. Luxemburgo.

Giuliano G (1986) Land use impacts of transportation investment: Highway and transit. In Gutierrez J, Gomez, G (1999) The impact of orbital motorways on intra-metropolitan accessibility: The case of Madrid's M-40. Journal of Transport Geography, 7: 1-15. 
Gutierrez J, Gómez G (1999) The impact of orbital motorways on intra-metropolitan accessibility: The case of Madrid's M-40. Journal of Transport Geography, 7: 1-15.

Gutiérrez J, Monzón A, Piñero J M (1994) Accesibilidad a los centros de actividad económica en España. Revista de Obras Públicas, 141 (3331): 34-49.

Gould P R (1969) Spatial Diffusion. Resource Paper 4. Association of American Geographers, Washington.

Haggett P (1975) Geography: a modern syntesis. Haper \& Row Publishers, New York.

Helling A (1996) Why we should care about intra-metropolitan accessibility and how we measure it. In Gutierrez J, Gomez, G (1999) The impact of orbital motorways on intra-metropolitan accessibility: The case of Madrid's M-40. Journal of Transport Geography, 7: 1-15.

Regional Policy - Inforegio. Urban Audit: What is the Urban Audit? (Consultada el 21 de octubre de 2008). Dirección URL: http://www.urbanaudit.org/help.aspx.

Izquierdo R, Monzón A (1992) La accesibilidad a las redes de transporte como instrumento de evaluación de la cohesión económica y social. TTC, 56: 33-56.

Monzón de Cáceres, A. (1988) Los indicadores de accesibilidad y su papel decisor en las inversiones en infraestructuras de transporte: aplicaciones en la Comunidad de Madrid. PhD Thesis. Universidad Politécnica de Madrid.

Pirie G H (1979) Measuring accesibility: a review and proposal. Enviroment and Planning A, 11 (3): 299-312.

Thrift N (1977). An introduction to time geography. Concepts and techniques in modern geography, Volumen 13, Norwich, Geo-Abstracts Ltd.

Vickerman (1995) Minority and Majority Groups. International Encyclopedia of Sociology, Vol: 2, Firzroy Dearborn Purblishers, Londres.

Zakaria T (1974) Urban transportation accessibility measures: modifications and uses. Traffic Quarterly, 28: 467-479. 